Document downloaded from:

http://hdl.handle.net/10251/98794

This paper must be cited as:

Meseguer Anastasio, JE.; Tavares De Araujo Cesariny Calafate, CM.; Juan-Carlos Cano (2017). On the Correlation Between Heart Rate and Driving Style in Real Driving Scenarios. Mobile Networks and Applications. 1-8. doi:10.1007/s11036-017-0833-x

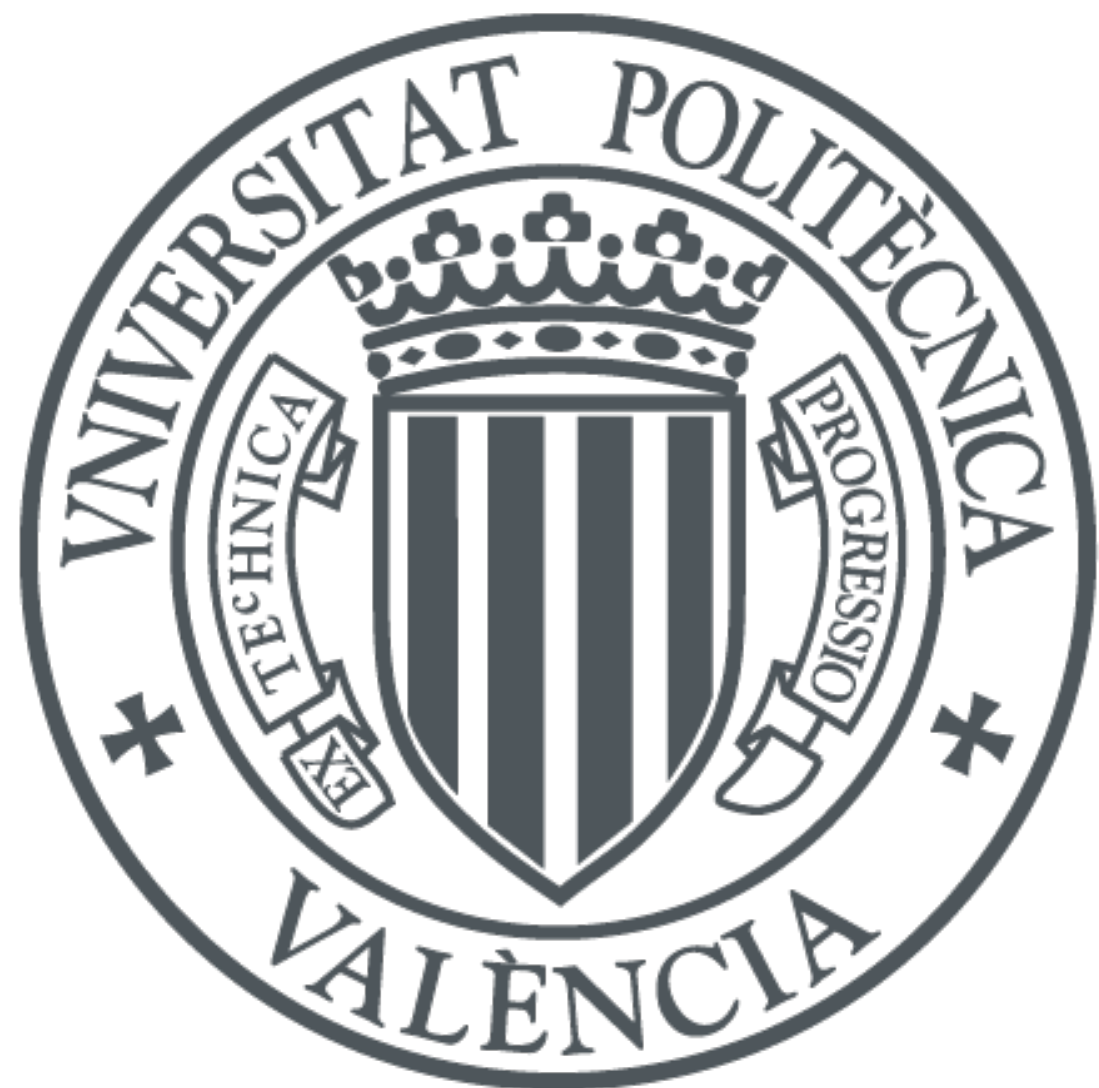

The final publication is available at

https://doi.org/10.1007/s11036-017-0833-x

Copyright Springer-Verlag

Additional Information 


\title{
On the correlation between heart rate and driving style in real driving scenarios
}

\author{
Javier E. Meseguer • Carlos T. Calafate • \\ Juan Carlos Cano
}

the date of receipt and acceptance should be inserted later

\begin{abstract}
Driving safety is of utmost importance in our society. The number of fatalities due to car accidents is still very high, and reducing this trend requires as much attention as possible. There are situations where the emotional conditions of drivers vary due to reasons beyond their control, or because they decide to change their driving style. Hence, we consider that such frequent situations deserve more scrutiny. In this work we addressed such issues by designing an Android application able to monitor in real-time both physiological data from the driver and diagnostic data from the vehicle (this data is obtained using an OBD-II connector) to study their correlation. Among the various non-invasive biomedical sensors available nowadays, we have adopted heart rate sensors, either packaged in belts or in smart watches. This allows studying the relationship between driving aggressiveness and heart rate. For our analysis we focused on fourteen different routes acounting a total driving time of 6 hours and 2 minutes which we have separated into three separate categories: urban, suburban, and highway routes. We analyzed the correlation between the heart rate and the driving style for each of the three groups. Our experiments show that the differences in terms of heart rate between quiet and aggressive behavior range between $2.5 \%$ and $3 \%$ beats per minute higher for the latter behaviour compared to the former.
\end{abstract}

Keywords Driving styles, Android smartphone, OBD-II, neural networks, driving behavior, heart rate, heart rate belt, eco-driving, consumption, road safety.

Department of Computer Engineering Universitat Politécnica de Valéncia Camino de Vera S/N, 46022, Spain

E-mail: jmesegue@upvnet.upv.es, \{calafate,jucano\}@disca.upv.es 


\section{Introduction}

It is known that prolonged or repeated stress, such as long traffic jams or driving on severely congested roads, is related to increased aggressiveness. In fact, authors like Gibson [1], Cohen [2], and Gravina et al. [3,4] have identified a potentially dangerous aggressiveness level as a result of driver stress. Thus, any method or system that can help at lowering the levels of aggressiveness when driving is welcome.

Recently, the smartphones have become ubiquitous, allowing to perform all types of tasks using both internal and external sensors connected wirelessly. In addition, access to the vehicles Electronic Control unit (ECU) was made possible through the availability of cheap On Board Diagnostics (OBD-II) devices [5-7], thus enabling a seamless vehicle-smartphone integration. Such integration paves the way for novel strategies in terms of driving style analysis, providing a detailed feedback about the driver actions upon the vehicle.

In this paper we focus our analysis on the correlation between driving style and driving stress measured as variations in the driver's heartbeart. To accomplish this goal we rely on our novel DrivingStyles architecture, which adopts data mining techniques and neural networks to analyze and generate a classification of the driving styles based on an analysis of the characteristics of the driver along the route followed. It ensures that drivers can be constantly aware of their level of aggressiveness and driving stress, and how this affects to their heart rate $[8,9]$. In a previous study [10], we developed a methodology to calculate, in real-time, the impact that the driving style will have on fuel consumption, and the environmental impact of spark ignition and diesel vehicles [11]. We demonstrated that an aggressive driving style increases the fuel consumption, as well as the emission of greenhouse gases [12-14].

In this paper we go one step forward and demonstrate that a more aggressive driving behavior also leads to a heart rate increase between $2.5 \%$ and $3 \%$ beats per minute with respect to a quiet behavior. The analysis has been carried out based on traces that correspond to 371 minutes of driving, taking 22290 direct samples (obtained from the vehicle's ECU and the driver's heart rate band), where the driver behavior and road types are analyzed. Our platform is able to assist drivers in correcting their bad driving habits, while offering helpful recommendations to improve fuel economy and driving safety. In terms of requirements, only off-the-shelf devices such as a mobile phone and an OBD-II device, along with a heart rate band or a smart watch, are required.

This paper is organized as follows: in the next section we present the DrivingStyles architecture. The method used for the analysis of the variables under study is presented in section 3. Experimental results are then presented in section 4. Finally, in section 5, we review the main conclusions and discuss future work. 


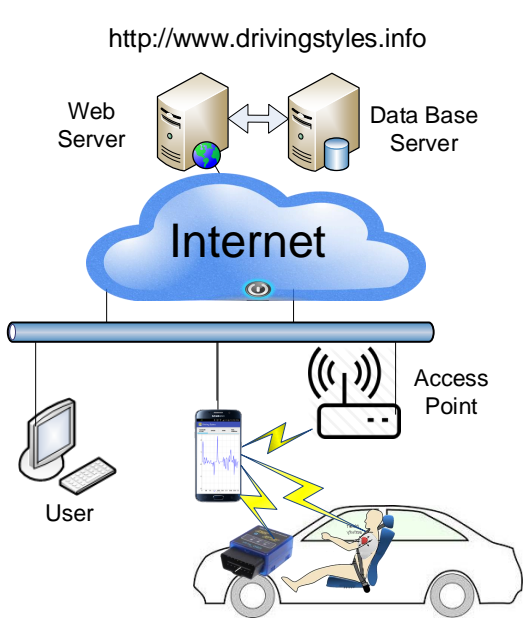

(a) Overview of the architecture

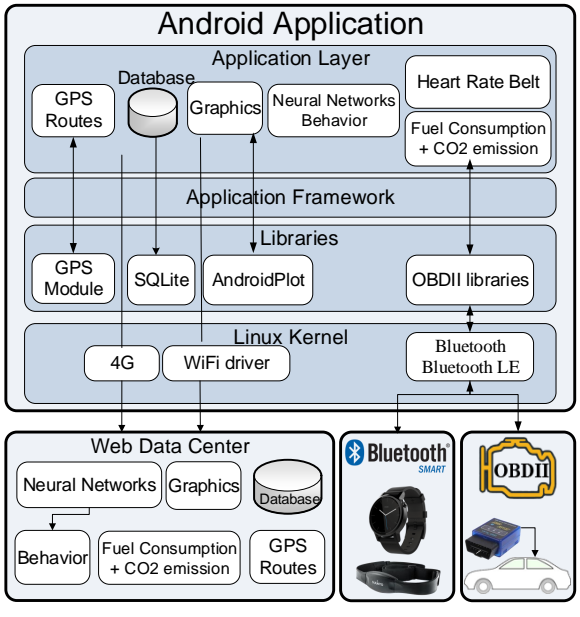

(b) Block diagram of the architecture

Fig. 1 System architecture of DrivingStyles: Overview and block diagram.

\section{General Overview of the DrivingStyles Architecture}

Our proposed architecture applies data mining techniques to generate a classification of the driving styles of users based on the analysis of their mobility traces using neural networks. Such classification is generated taking into consideration the characteristics of each route, such as whether it is urban, suburban, or highway. To achieve the overall objective, the system is structured around the following two elements:

1. An application for Android-based smartphones which is responsible for collecting data from the car and the driver's heart rate band or smart watch, and that also analyzes routes and driver behavior using neural networks.

2. A cloud-based datacenter to collect large data sets sent by different users concurrently. Subsequently, these data are analyzed using data mining and expert systems, in order to generate useful information.

\subsection{Android Application}

Using an OBD-II Bluetooth interface, the Android application (see figure 1.b) collects information such as speed, acceleration, engine revolutions per minute, throttle position, and the vehicles geographic position. It also obtains information from a wearable heart rate monitor, chest belts, and smart watches. This information is analyzed on the device itself, performing the analysis of driver behavior, and road type (using neural networks), instantaneus fuel consumption, greenhouse gas emissions, and heart rate measurement. 
We then provide feedback from the device to the user in a way that, when the application detects high levels of aggressiveness (above a certain threshold), the device generates an acoustic signal to alert the driver. Furthermore, if the user has a wearable device such as a smart watch, it is notified through a similar vibration event.

Eventually, the user uploads the route data to the remote data center for a more comprehensive analysis. For more detailed information please refer to $[15,10]$.

\subsection{Data Center}

Our data center ${ }^{1}$ is able to collect large data sets sent by different users (see figure 1.a). It allows users and administrators to access data about routes and provides per-user statistics. To implement this component, we have selected open source software such as Apache HTTP, and Joomla as the content management system (CMS).

Basically, the server receives data sent from the Android application of each user, and it provides functionality to work with User, Routes, and Statistics. Once the user is logged in, he is asked to record a number of important data, especially for future data mining studies. The most relevant items are sex, age, and other details concerning the vehicle used: car manufacturer, model, and fuel type, among others.

\section{Research Strategy and Methodology}

We now present the methodology we have followed in order to correlate driving aggresiveness and driver heart rate by using the data provided by our DrivingStyles architecture.

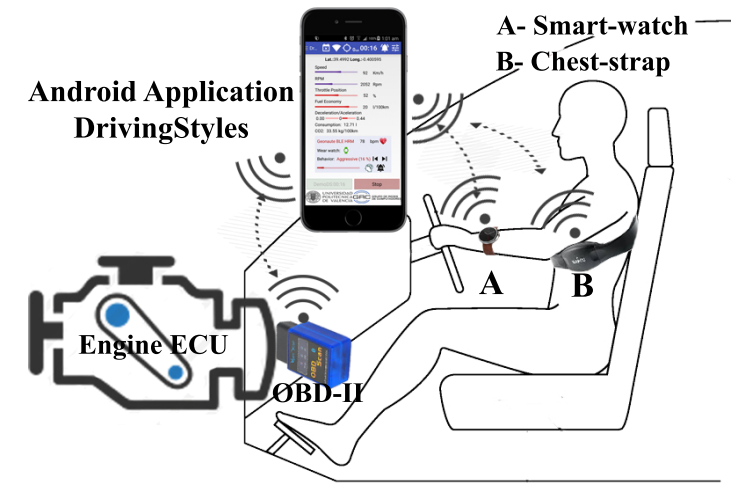

Fig. 2 On Board schema components of DrivingStyles instrumentation.

\footnotetext{
1 http://www.drivingstyles.info
} 


\subsection{Participant}

In this study we have relied on fourteen routes (total of 6 hours and 11 minutes) of varying duration carried out by a single subject in completely different environments (urban, suburban or highway), and for different road weather conditions (rainy, sunny, and cloudy). This scenario diversity allowed us to analyze the system reliability under in different situations [16], thus achieving a higher confidence on results.

The data reported in the present study were collected from a 36 year-old male driver, without heart deseases, and whose heart rate while at rest lies between $70 \mathrm{bpm}$ (beats per minute) and $75 \mathrm{bpm}$. The driver was equipped with an Android device running our DrivingStyles application, and a heart rate band (brand Geonaute, although any other compatible band could be used as well) attached to the driver's chest.

\subsection{OBD-II Car Instrument}

The vehicle used for testing is a gasoline KIA model with manual transmission. It was instrumented with an interface compatible with the OBD-II standard [6,5], available since 1994 [7], and that has recently become an enabling technology for in-vehicle applications due to the appearance of Bluetooth OBD-II connectors $[17,7]$. These connectors enable a transparent connectivity between the mobile device and the vehicles ECU, see figure 2.

\subsection{Heart Rate Monitor (HRM)}

Regarding heart rate monitoring (HRM) devices, there are mainly two types on the market: the smartwatch (or other wrist band), and the chest strap. Notice that, due to technological restrictions, smartwatch models tend to be less accurate than chest-strap HRMs. Tests were conducted with different models of both types. The first devices used were wrist devices, including the Motorola 360 smartwatch. In this model, the back of the watch hosts the heart rate sensor. Despite using oximeter-based technology for pulse measurement, the sampling frequency of the smart watch was too low for our purposes, and, in combination with the high battery consumption when the heart rate measurement function is activated, made us disregard this device from the beginning. So, we opted for the heart rate belt device instead.

As we can see in figure 3, the Android app displays the heart rate in realtime, as well as a map representation of the heart rate compared with the mean value for the part of the route completed so far, being red if it is higher than the mean, and green otherwise. 


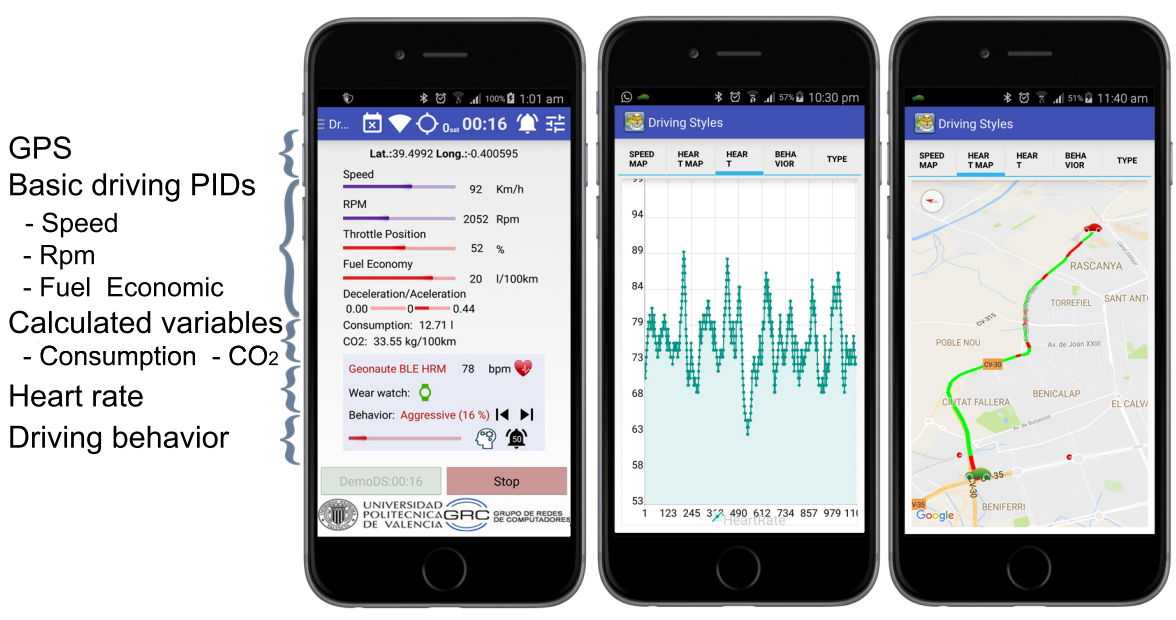

Fig. 3 Snapshots of the main screen and the heart rate module for the Android application.

\subsection{Measurement Results}

This study was carried out with a total of fourteen routes (nine urban routes, two suburban routes, and three highway routes), and a total driving time of 6 hours and 11 minutes (371 minutes). Regarding the heart rate, 22290 direct samples (one sample every second) have been obtained. Also, 4458 driving behavior measures calculated by the neural network have been used in the test, reflecting the behavior of the driver at measurement time (behavior analysis is performed with data taken from a time window starting 5 seconds before performing the calculation).

Table 1 we presents details about the different routes taken, including the total number of samples and the total driving time for each route, as well as the mean heart rate. Since these data refer to real everyday life situations, urban routes are typically short-lived, while highway routes are much longer, having suburban routes an intermediate duration.

In Section 4 we proceed by analysing the correlation between driving behavior and the driver's heart rate.

\section{Experimental Results and Evaluation}

In general we can assume that urban drivers can be exposed to higher levels of stress during rush-hours [18]. Similarly, the sparsest traffic conditions can be found in scenarios such as countryside roads, while driving on highways, or in sparsely populated areas. Hence, these two conditions should represent the far ends that we should find in the routes under analysis. 
Table 1 Number of samples, time, and mean heart rate value for the different routes under analysis.

\begin{tabular}{lcccc}
\hline Route Type & Number Routes & Samples & Total Time & Heart Rate Mean \\
\hline Urban & 9 & 4291 & $1 h 03^{\prime} 39^{\prime \prime}$ & 72.95 \\
Suburban & 2 & 4353 & $1 h 12^{\prime} 33^{\prime \prime}$ & 77.77 \\
Highway & 3 & 13664 & $3 h 47^{\prime} 18^{\prime \prime}$ & 78.85 \\
\hline
\end{tabular}

Table 2 Slope-intercept form equation of urban, suburban, and highway routes.

\begin{tabular}{lcc}
\hline Behavior Quiet-Normal-Aggressive & $y$ & $R$ \\
\hline Urban (9 routes) & $3.054 x+70.221$ & 0.103 \\
Suburban (2 routes) & $1.727 x+71.603$ & 0.069 \\
Highway (3 routes) & $8.474 x+73.552$ & 0.416 \\
\hline
\end{tabular}

Three case studies are analyzed: the driver moving in an urban scenario (9 routes), the same driver moving in a suburban scenario (2 routes), and the driver circulating in a highway (3 routes). Overall, total time of these case studies adds up to 6 hours and 11 minutes.

In all cases, we obtain through linear regression the approximation that better describes the correlation between both data sets. A positive gradient shall validate our assumption of a positive correlation between driver behavior and heart rate, as intended (see table 2).

\subsection{Driving tests in urban areas}

The combined data from all urban routes adds up to 71 minutes and 22 seconds (4282 seconds) of urban driving, which corresponds to a total of 857 behavior samples calculated by the system.

Table 3 Mean, standard deviation, and range of route time, speed, and heart rate of urban routes.

\begin{tabular}{lccc}
\hline & Mean & SD & Range \\
\hline Routes time $($ minutes $)$ & 7.75 & 2.64 & $3.11-13.10$ \\
Speed $(\mathrm{km} / \mathrm{h})$ & 31.14 & 24.85 & $0-84$ \\
Heart rate $(\mathrm{bpm})$ & 71.92 & 6.08 & $60-89$ \\
\hline
\end{tabular}

By performing linear regression we find that equation $3.054 x+70.221$ is the corresponding regression line in the slope-intercept form, where the slope is given by $m=3.054$, and the y-intercept by $b=70.221$ (See table 2 for a slope comparison against the other two studies). As it can be observed, the intended correlation between driving styles and heart rate $R$ is 0,103 in the urban scenario. The correlation value is relatively low when compared to the 


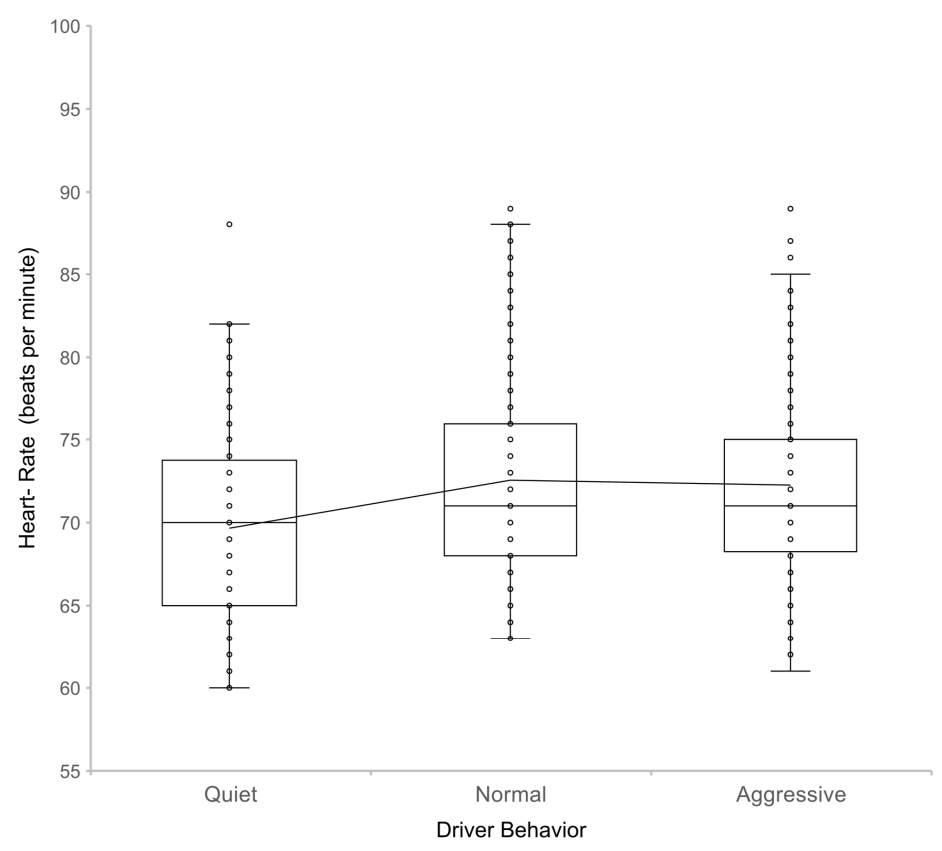

Fig. 4 Box and wisker plot of heart rate vs driving behavior in urban routes.

result for highway routes (where $R=0,416$ ), but it is significantly greater than the correlation obtained in suburban driving (where $R=0,069$ ), as shown in the following sections.

The slope of the line also follows this trend: the slope of the equation for highway driving is the largest $(m=8,474)$, followed by the urban cases where $m=3,054$.

These results were mostly expected, since driving in urban areas is more stressful in most cases than suburban driving by requiring more driver attention and greater control due to the presence of other drivers. Surprisingly, the greater slope was achieved for the equation describing the highway scenario. Apriori, highway driving is less stressful, but we should point out that the total time of the route is much higher, resulting in driver tiredness, as discussed in more detail in section 4.3.

We find that the difference in heart rate between quiet and aggressive behavior is $3,05 \%$, about three beats per minute, as shown in figure 4 .

\subsection{Driving Tests in Suburban areas}

In this second part of the analysis, we study two specific routes chosen from the set of fourteen routes analyzed, specifically those corresponding to suburban driving. Notice that such discrimination is possible since the DrivingStyles 
platform, in addition to analyzing the driver behavior, is also able to compute the route type.

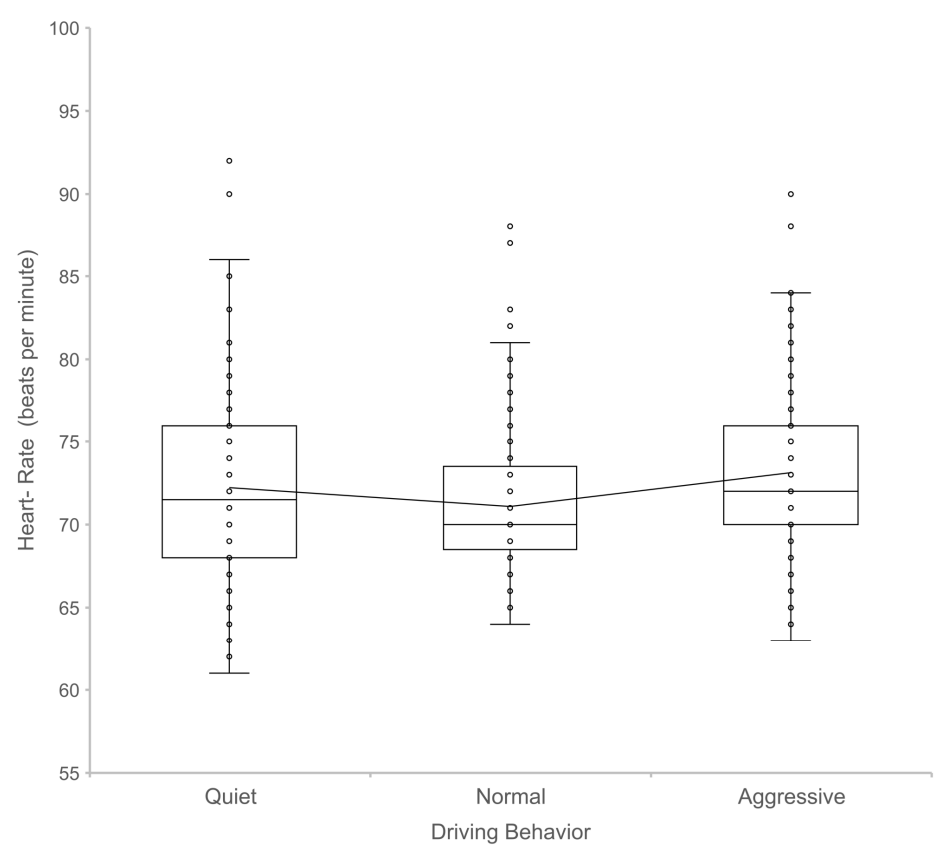

Fig. 5 Box and wisker plot of heart rate vs driving behavior in suburban routes.

The two analyzed routes have a total duration of 82 minutes and 33 seconds, circulating at a mean speed of $34.26 \mathrm{~km} / \mathrm{h}$ and a maximum speed of $112 \mathrm{~km} / \mathrm{h}$, covering a total of $28.25 \mathrm{~km}$; this corresponds to 4353 driving behavior-type samples, also including data about the driver's heart rate that we use for statistical analysis.

Table 4 Mean, standard deviation, and range of route time, speed, and heart rate of suburban routes.

\begin{tabular}{lccc}
\hline & Mean & SD & Range \\
\hline Routes time $($ minutes $)$ & 35.96 & 3.56 & $33.45-38.48$ \\
Speed $(\mathrm{km} / \mathrm{h})$ & 34.26 & 26.51 & $0-112$ \\
Heart rate $(\mathrm{bpm})$ & 72.39 & 5.93 & $61-92$ \\
\hline
\end{tabular}

The results are not too noticeable compared to the previous section (urban driving, see section 4.1) and the next section (highway driving, see section 4.3). In this case we find that, when the driver has a normal behavior, the heart 
rate often becomes lower with respect to a quiet behavior, as can be observed clearly in the box and whisker plot (see figure 5) which displays the differences in terms of heart rate between quiet, normal, and aggressive driving behavior. This means that differences between quiet and normal behaviour are not too significant, and that, at times, quiet driving is not a driver decision, but it is rather forced due to external conditions (e.g. traffic jam), which can cause stress. For this test subject, though, aggressive driving provoked a slightly increased heart rate.

\subsection{Driving Tests in Highways}

In this third part of the analysis, we evaluate three routes with a total duration of 3 hour and 46 minutes, and where the mean speed is of $100.18 \mathrm{~km} / \mathrm{h}$, the maximum speed is of $134 \mathrm{~km} / \mathrm{h}$, and the total distance covered is $394 \mathrm{~km}$. In total, we have 13664 driving behavior-type samples, also including data about the driver's heart rate that we use in the statistical analysis. The most relevant characteristics of the 3 highway routes can be seen in table 5

Table 5 General characteristics of highway routes.

\begin{tabular}{lccccc}
\hline & Total Time & Start Time & Arrival Time & Max Speed & Mean Speed \\
\hline Highway 1 & 81.44 & $09: 45: 33$ & $11: 07: 18$ & $134 \mathrm{~km} / \mathrm{h}$ & $96.99 \mathrm{~km} / \mathrm{h}$ \\
Highway 2 & 79.32 & $18: 35: 25$ & $19: 54: 58$ & $120 \mathrm{~km} / \mathrm{h}$ & $100.67 \mathrm{~km} / \mathrm{h}$ \\
Highway 3 & 74.42 & $16: 16: 11$ & $17: 30: 55$ & $119 \mathrm{~km} / \mathrm{h}$ & $103.16 \mathrm{~km} / \mathrm{h}$ \\
\hline
\end{tabular}

Results show that the linear trendline remains clearly positive, being the slope value $m=8.474$, and the coefficient of determination $R=0.416$. This correlation coefficient indicates that there is a clear lineal relationship between aggressive behavior and heart rate. Highway driving is the one that most clearly shows the impact of driving aggressiveness on heart rate values. It should also be denoted that the routes studied in this section are all long routes, being able to produce an increased heart rate due to the fatigue caused by prolonged driving.

Table 6 Mean, standard deviation, and range of route time, speed, and heart rate of highway routes.

\begin{tabular}{lccc}
\hline & Mean & SD & Range \\
\hline Routes time (minutes) & 75.33 & 3.05 & $77.03 .45-78.12$ \\
Speed $(\mathrm{km} / \mathrm{h})$ & 100.18 & 22.53 & $0-134$ \\
Heart rate $(\mathrm{bpm})$ & 78.46 & 6.19 & $60-114$ \\
\hline
\end{tabular}




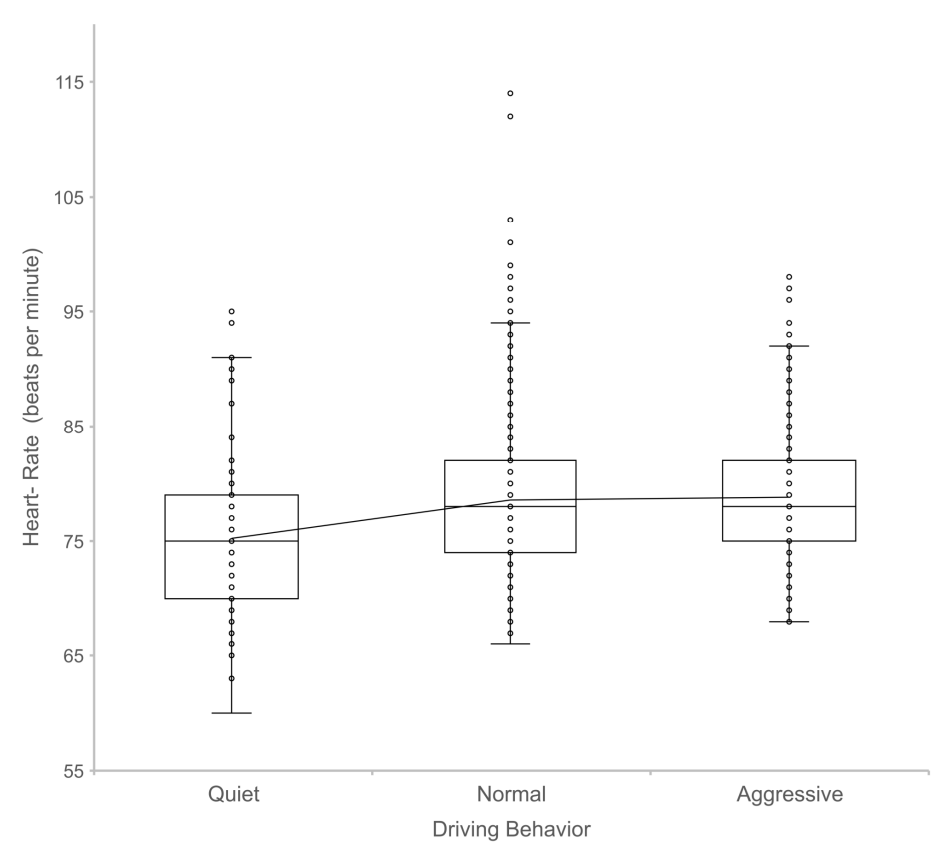

Fig. 6 Box-and-whisker plot of heart rate vs. driving behavior in highway routes.

We now proceed to study these three routes in more detail, highlighting the particularities of each of them:

1. In one of the highway routes the driver had an unexpected stop requiring getting out of the vehicle, which affected his heart rate, as we can see in figure 7. Specifically, the heart rate increased until reaching $117 \mathrm{bpm}$, taking several minutes to regain a normal heart rate. However, as we can observe in the same chart, the system does not detect any change in the driving behavior. Another thing that can be observed in the chart is that the system detects a quiet behavior at the end of the route because the driver is approaching the end of his trip. In fact, it can clearly be observed how the heart rate decreases slowly, being that the DrivingStyles system detects the behavior becoming quiet, and graphically represents these final points in green.

2. In the second long-duration route (see figure 8 ) it can be seen how the heart rate decreases gradually due to the driver sleepiness, and at a certain moment the driver decides to turn on loud music to get awake. The chart shows how the heart rate has a peak and reaches $91 \mathrm{bpm}$, but, as can be seen, the system does not detect a driver behavior change. Thus, this example highlights that indeed loud music can help at increasing the heart rate, preventing sleepiness and reducing the chances of having an accident, without necessarily causing changes in the driving behavior. 
3. In the last studied route, we can see that there is a period of the route where the driver is clearly stressed and altered; the system detects that it has an aggressive behavior during 17 minutes, as can be seen in figure 9 . The driver then calms down and the heart rate normalizes for the rest of the trip.

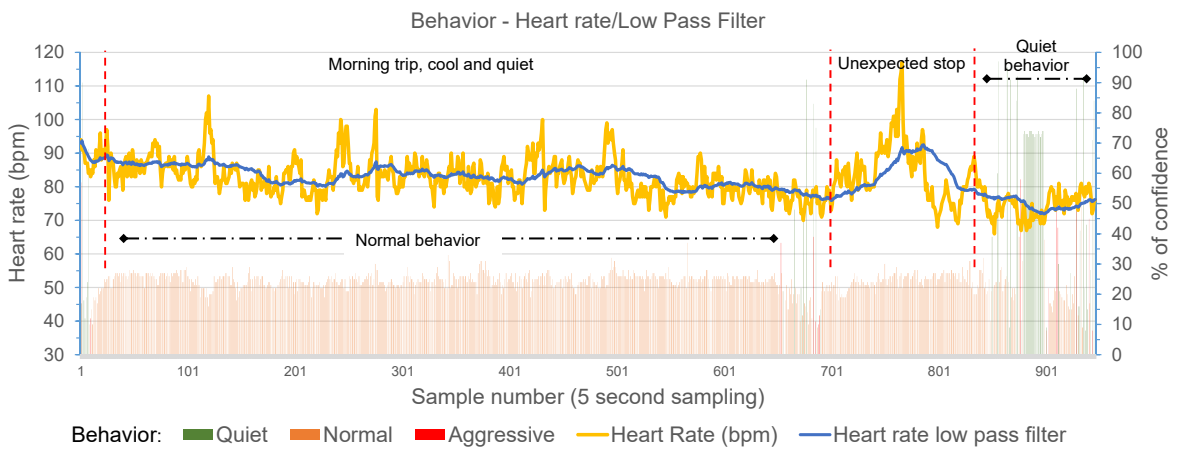

Fig. 7 Highway 1:Heart rate increases to $117 \mathrm{bpm}$ due to an unexpected stop.

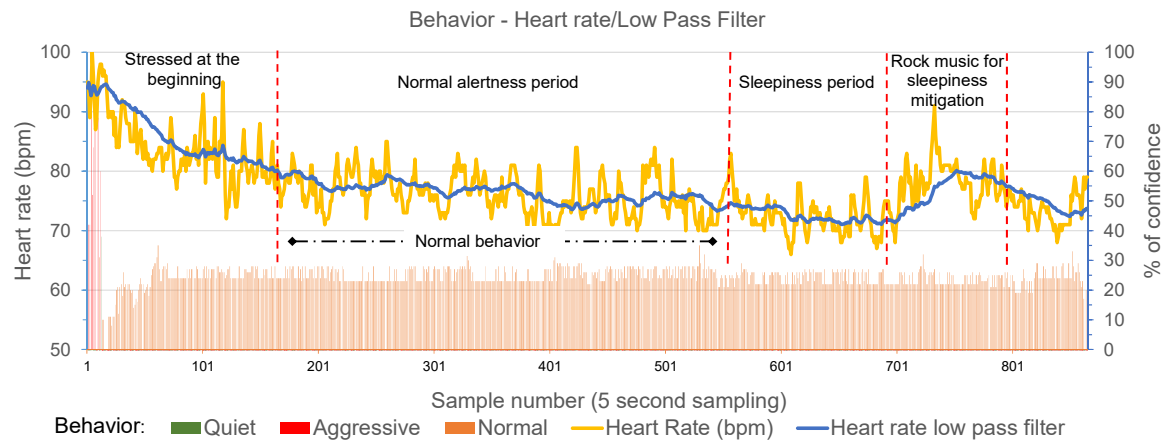

Fig. 8 Highway 2: Heart rate decreases gradually due to driver sleepiness. 


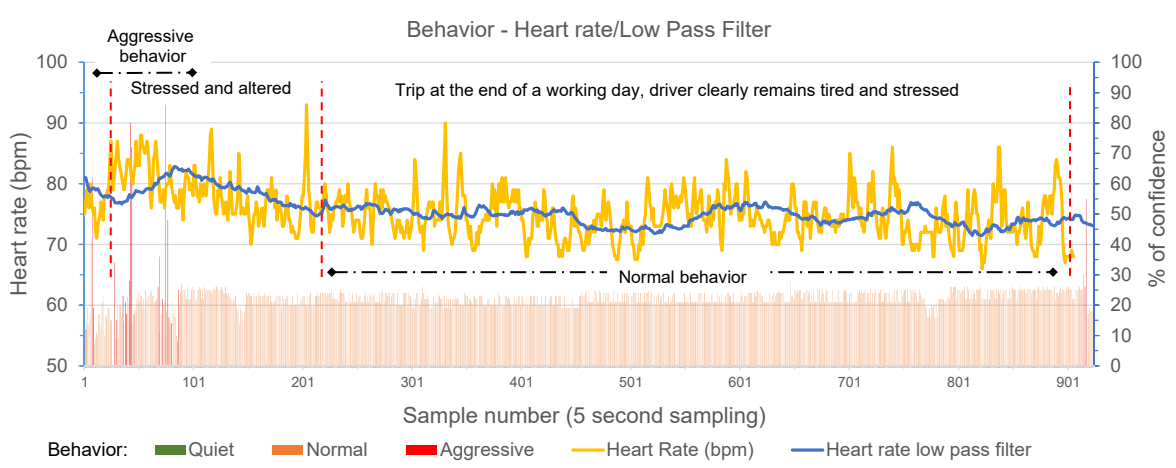

Fig. 9 Highway 3: Route where the driver is clearly stressed and altered.

\section{Conclusions and Future Work}

In this paper we studied the correlation between driver heart rate and driver behavior. We based our study on the use of our novel DrivingStyles architecture, that combines new technologies such as smartphones and wearable body sensors with modern software implementations of artificial neural networks.

The results of the present study indicated that aggressive driving caused an increase in the heart rate, being able to rise it by up to $2,5 \%$ and $3 \%$ beats per minute. Based on our experimental results, we have reached the conclusion that the difference in terms of heart rate between a quiet and aggressive behavior ean become very noticeable. In statistical terms, we also found that, as the number of samples increases, the correlation between the driver behavior and heart rate is significant. This expers since increasing the number of routes whose behavior is largely quiet makes the percentage of values with an aggressive behavior to decrease, i.e., an urban route that causes stress and aggressiveness will get closer to our results that a long highway route where the stress is non-existent or scarce, although fatigue can affect driving behavior. Since this study has been conducted taking a middle-aged male subject as reference, in future works we will expand the scope to more drivers of different ages, which will help at covering a wider range of possible cases. This will allow us to study the differences between various age segments, as well as to differentiate driving aggressiveness and heart rate based on the driver's gender.

\section{Acknowledgments}

This work was partially supported by the Ministerio de Economía y Competitividad, Programa Estatal de Investigación, Desarrollo e Innovación Orientada a los Retos de la Sociedad, Proyectos I+D+I 2014, Spain, under Grant TEC2014-52690-R. 


\section{References}

1. Gibson PM and Wiesenthal DL., The Driving Vengeance Questionnaire (DVQ): the development of a scale to measure deviant drivers, LaMarsh Research Programme Report Series, No. 54 June 1996, Volume, page numbers (year)

2. Cohen S, After effects of stress on human performance and social behavior: a review of research and theory, Psychol Bull 88:82108, 1980

3. Raffaele Gravina and Giancarlo Fortino,Automatic Methods for the Detection of Accelerative Cardiac Defense Response,IEEE Transactions On Affective Computing, Vol. 7, NO. 3,2016

4. Alessandro Andreoli et al.,SPINE-HRV: A BSN-Based Toolkit for Heart Rate Variability Analysis in the Time-Domain,Wearable and Autonomous Systems, LNEE 75, pp. 369389. A. Lay-Ekuakille et al.

5. Keyword Protocol 2000,International Organization for Standardization,1999

6. ISO 9141-2:1994/Amd 1:1996,1196,International Organization for Standardization,1996

7. M Awais Khan and Aii Raza,Development of an On-board Diagnostic (OBD) Kit for Troubleshooting of Compliant Vehicle,Emerging Technologies (ICET), 2013 IEEE 9th International Conference,2013

8. Haykin, S.,Neural Networks: A Comprehen-sive Foundation, Macmillan, p. 2.,1994

9. Hecht-Nielsen, R., Theory of the backpropagation neural network - Neural Networks,IJCNN., International Joint Conference,1989

10. Meseguer, J.E. and Calafate, C.T. and Cano, J.C. and Manzoni, P., Assessing the Impact of Driving Behavior on Instantaneous Fuel Consumption, IEEE 12th Consumer Communications and Networking Conference (CCNC) (CCNC 2015)

11. Kuhler, M. and Kartens, D., Improved driving cycle for testing automotive exhaust emissions, SAE Technical Paper Series 780650., 1978

12. Johansson H. et al.,Impact of EcoDriving on emissions., International Scientific Symposium on Transport and Air Pollution, Avignon, France., 2003

13. Ahn K, Estimating vehicle fuel consumption and emissions based on instantaneous speed and acceleration levels., Journal of Transportation Engineering., 2002

14. Rakha, H., Simple Comprehensive Fuel Consumption and CO2 Emission Model based on Instantaneous Vehicle Power., Transportation Research Board Annual Meeting. Washington D.C., 2011

15. Meseguer, J.E. and Calafate, C.T. and Cano, J.C. and Manzoni, P., DrivingStyles: a smartphone application to assess driver behavior, 18th IEEE symposium on Computers and Communications (ISCC 2013), 2013

16. A. Riener, Subliminal Persuasion and Its Potential for Driver Behavior Adaptation, IEEE Transactions On Intelligent Transportation, pp. 1-10 ., 2012

17. ELM327DS. OBD to RS232 Interpreter, Elm Electronics Circuits, 2010

18. Timo Lajunen;Dianne Parker;Heikki Summala, Does traffic congestion increase driver aggression?, Transportation Research Part F 2 225-236, 1999 